\title{
Enhanced flow rate by the concentration mechanism of Tetris particles when discharged from a hopper with an obstacle
}

\author{
Guo-Jie Jason Gao, ${ }^{1}$, Fu-Ling Yang, ${ }^{2}$ Michael C. Holcomb, ${ }^{3}$ and Jerzy Blawzdziewicz ${ }^{4}, 5$ \\ ${ }^{1}$ Department of Mathematical and Systems Engineering, \\ Shizuoka University, Hamamatsu, Shizuoka 432-8561, Japan \\ ${ }^{2}$ Department of Mechanical Engineering, National Taiwan University, Taipei 10617, Taiwan \\ ${ }^{3}$ Department of Physics and Geosciences, Angelo State University, San Angelo, TX 76909-0904, USA \\ ${ }^{4}$ Department of Physics, Texas Tech University, Lubbock, TX 79409-1051, USA \\ ${ }^{5}$ Department of Mechanical Engineering, Texas Tech University, Lubbock, TX 79409-1021, USA
}

(Dated: May 14, 2021)

\begin{abstract}
We apply a holistic 2D Tetris-like model, where particles move based on prescribed rules, to investigate the flow rate enhancement from a hopper. This phenomenon was originally reported in the literature as a feature of placing an obstacle at an optimal location near the exit of a hopper discharging athermal granular particles under gravity. We find that this phenomenon is limited to a system of sufficiently many particles. In addition to the waiting room effect, another mechanism able to explain and create the flow rate enhancement is the concentration mechanism of particles on their way to reaching the hopper exit after passing the obstacle. We elucidate the concentration mechanism by decomposing the flow rate into its constituent variables: the local area packing fraction $\phi_{l}^{E}$ and the averaged particle velocity $v_{y}^{E}$ at the hopper exit. In comparison to the case without an obstacle, our results show that an optimally placed obstacle can create a net flow rate enhancement of relatively weakly driven particles, caused by the exit-bottleneck coupling if $\phi_{l}^{E}>\phi_{o}^{c}$, where $\phi_{o}^{c}$ is a characteristic area packing fraction marking a transition from fast to slow flow regimes of Tetris particles. Utilizing the concentration mechanism by artificially guiding particles into the central sparse space under the obstacle or narrowing the hopper exit angle under the obstacle, we can create a man-made flow rate peak of relatively strongly-driven particles that initially exhibit no flow rate peak. Additionally, the enhanced flow rate can be maximized by an optimal obstacle shape, particle acceleration rate towards the hopper exit, or exit geometry of the hopper.
\end{abstract}

\section{INTRODUCTION}

Both experimentally and numerically, placing an obstacle at an optimal distance away from the exit of a hopper has been shown to enhance the gravity-driven granular hopper flow rate on the order of ten percent [16. This strategy has been shown to be effective not only on passive granular particles but also on self-governing species [7-11]. One of the possible explanations for the enhanced flow rate is the waiting room effect, wherein particles are slowed down by the obstacle and then accelerate within the void underneath it on their way towards the hopper exit [1, 3, 4, 6, 8]. However, some studies are either unable to reproduce this phenomenon [12, 13, or able to reproduce it even under conditions in which the void space below the obstacle has been eliminated through the introduction of a special obstacle shape that diminishes the waiting room effect [6]. Conventional experiments and numerical approaches, governed by Newtonian dynamics, contain multiple competing mechanisms such as interparticle collaborative motion and particle acceleration due to gravity; these cannot be easily decomposed and inspected separately. To isolate the roles of these competing mechanisms, a more primitive dynamic model is needed.

\footnotetext{
* gao@shizuoka.ac.jp
}

In our previous studies, we showed that the interparticle friction, particle dispersity, and obstacle geometry are not directly responsible for the enhanced flow rate [14. We then proposed a 2D Tetris-like model, where particles move according to prescribed rules rather than in response to forces in order to switch off interparticle collaborative motion. Using our model, we still observed the enhanced flow rate; therefore, the collaborative motion of particles via Newtonian dynamics is also not the key mechanism 14, 15. Another more simplified Tetris-like model, where particles can only move diagonally, was proposed to study the packing behavior of granular materials under vibration [16. In contrast to reductionist models, such as discrete element methods that preserve enough details to quantitatively reproduce an aimed physical phenomenon, Tetris-like models are holistic and focus on similarities between different nonequilibrium systems containing animate or inanimate discrete particles. The results of our model suggest that the concentration of particles arriving at the hopper exit is essential to the observed flow rate peak, as the local area packing fraction of particles $\phi_{l}$ (defined in the cited reference [14] and again in Sec. IIIB increases near the hopper exit and can become larger than its value near the obstacle. In contrast, we do not observe the same pattern of $\phi_{l}$ variation in a system that exhibits no flow rate peak.

Following this finding, this work further uses the 2D Tetris-like model to explore how the flow rate is influ- 
enced by the number of particles in the hopper; the concentration behavior of particles below the obstacle; and the waiting room effect, as it relates to obstacle geometry, particle acceleration, and exit geometry of the hopper. We find that the flow rate peaking phenomenon is limited to a system of sufficiently many particles, and that an obstacle in a hopper containing too few particles only reduces its flow rate. In addition, placing an obstacle in a hopper of sufficiently many particles can reduce the particle area packing fraction more than without an obstacle and, therefore, particles move faster. The net effect is two downstream particle flows concentrating at the hopper exit with an area packing fraction able to be greater than a characteristic value, which triggers an exit-bottleneck coupling. This mechanism is directly responsible for the flow rate peaking phenomenon if particles are relatively weakly-driven and the obstacle is optimally placed. Moreover, we utilize the concentration mechanism to prove that a flow rate enhancement can be generated in a system discharging relatively stronglydriven particles that originally exhibits no flow rate peak. This can be accomplished by artificially increasing the horizontal driving strength asymmetrically of the particle flowing down stream of the obstacle, or by narrowing the hopper exit angle under the obstacle. Finally, we evaluate the waiting room effect and show the existence of an optimal obstacle geometry, particle acceleration rate towards the hopper exit, and exit geometry can maximize or create an enhanced flow rate.

Below we elaborate on our Tetris-like model which generates the probability-driven hopper flow in section II. followed by quantitative investigations of the hopper flow rates under different conditions in section III with discussions. We conclude our study in section IV.

\section{THE TETRIS-LIKE MODEL}

To study the simplest 2D granular hopper flow without invoking Newtonian dynamics, we propose a model, named after the video game Tetris, where objects fall one at a time following some prescribed rules within a confined space. Per position-update cycle in our model, each particle $i$ of uniform diameter $d$ attempts to move exactly once from its current $x$ and $y$ positions $\left(x_{i}^{\text {old }}, y_{i}^{\text {old }}\right)$ to $\left(x_{i}^{n e w}, y_{i}^{\text {new }}\right)$, specified by

$$
\begin{aligned}
& x_{i}^{\text {new }}=x_{i}^{\text {old }}+N_{x}\left(0, \alpha_{x} \sigma\right) \\
& y_{i}^{\text {new }}=y_{i}^{\text {old }}-\left|N_{y}\left(0, \alpha_{y} \sigma\right)\right| r_{s}^{n_{i}^{s}}
\end{aligned}
$$

where $N_{x}$ and $N_{y}$ are normal distribution functions having zero means and standard deviations $\alpha_{x} \sigma$ and $\alpha_{y} \sigma$, and specify displacements of particle $i$. The two independent control parameters $\alpha_{x}$ and $\alpha_{y}$ determine the driving strengths in the horizontal $x$ and vertical $y$ directions, respectively. The absolute value about $N_{y}$ guarantees that a particle always moves in only one direction towards the hopper exit. We chose a moderate $\sigma=0.05 d$ so that (a)

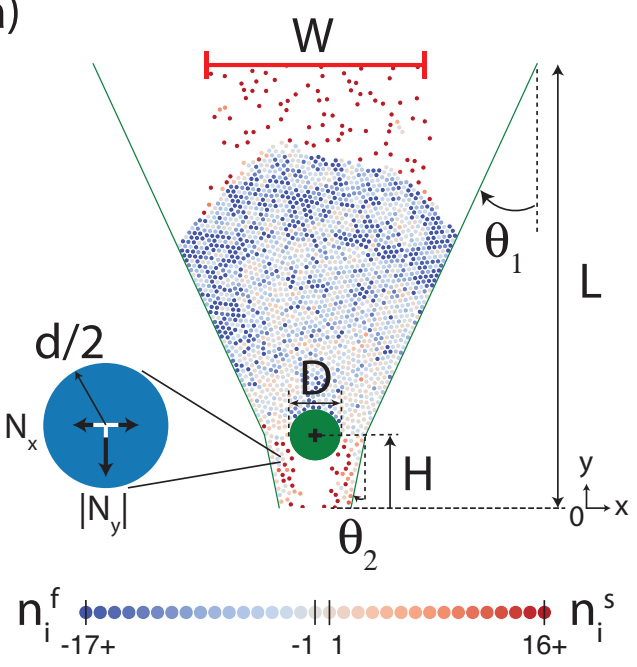

(b)
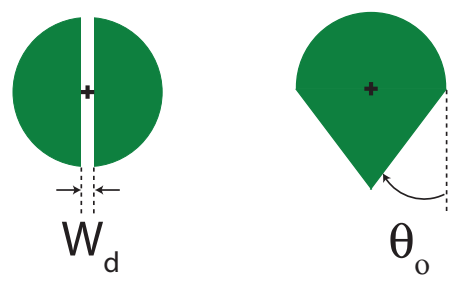

FIG. 1. (Color online) (a) A snapshot of the system setup. A symmetric hopper (green lines) with equal height and topwidth $L$, a hopper angle $\theta_{1}$, and an exit angle $\theta_{2}$ contains a round obstacle (green circle) of diameter $D$, placed along its centerline at a height $\mathrm{H}$ above its exit. Particles discharged from the hopper reenter it from its top boundary with their $x$ positions randomized in between $W=0.5 \mathrm{~L}$. The discrete redblue colors represent the value of $n_{i}^{s}>0$ or $n_{i}^{f}<0$, recording the position-update history of consecutive successes or failures. (b) The other two kinds of obstacles, a hollow circle and a semicircle-triangle, used in the study. Their circular parts have the same diameter $D$, and they are also located at a height $H$ above the hopper exit, measured from their centers (cross marks).

on average particles exit the hopper containing no obstacle with their trajectories parallel to the hopper walls [15]. A move attempt is only realized if it creates no overlap between any object in the system. Otherwise, the move attempt is rejected and the attempted particle stays still. Which particle moves first is determined by a random sequence, regenerated at the beginning of each position-update cycle. Additionally, each particle $i$ remembers its position-update history, recorded as a monotonically increasing number $n_{i}^{s}>0$ or decreasing number $n_{i}^{f}<0$ for consecutive successful or failed attempts. Whenever one parameter becomes nonzero, the other is reset to zero. The speed-up rate $r_{s} \geq 1$ in the vertical $y$ direction mimics the effect of particle acceleration due to gravity during free fall. A particle that successfully updates its position $n_{i}^{s}$ times can attempt a 
longer jump due to acceleration by a factor of $r_{s}{ }^{n_{i}^{s}}$ during the next position-update cycle. We do not include $n_{i}^{f}$ in Eqn. 1 because it is intrinsically related to particle rebounding behavior, which is not allowed in the current model. The Tetris-like model can also be viewed as a $2 \mathrm{D}$ cellular automaton in a sense that the size of a cell, surrounded by a circular excluding zone of diameter $d$, is infinitesimally small down to the machine precision. Additional details about the Tetris-like model can be found in our previous studies [14, 15.

The geometrically symmetric hopper, measuring $L=$ $83 d$ in height with a fixed hopper angle $\theta_{1}=0.4325(\mathrm{rad})$ and a changeable exit angle $\theta_{2}$, contains $N$ randomly placed particles at the beginning of each simulation. The the hopper's orifice size is a function of $\theta_{1}$ and $\theta_{2}$. In all figures except Fig. 13 and Fig. 14. $\theta_{2}=\theta_{1}$, which gives an orifice width of about $6.366 d$. In Fig. 13 and Fig. 14. the orifice size decreases with increasing $\theta_{2}$, where the maximum value of $\theta_{2}=0.5$ corresponds to the minimum orifice size of about $4.061 d$. To conserve the total number of particles within the system, $N$, a particle coming out of the hopper from its exit will reenter it from above with the particle's new $x$ position randomized inbetween $W=[-L / 4, L / 4]$, as shown by a snapshot in Fig. 1(a). After a simulation reaches its steady state, we measure the flow rate $J_{o}$ in terms of the average number of particles passing the orifice of the hopper containing no obstacle per position-update cycle. Similarly, we measure $J_{a}$ when the hopper contains a circular obstacle or an obstacle with a semicircular component of diameter $D=0.112 L$ whose center is placed along the symmetric axis of the hopper at a height $H$ above its exit. We use obstacles of different shapes to study the effect of the obstacle geometry on the flow rate, such as an obstacle with a hollow duct of width $W_{d}$ or another one composed of a semicircle top and isosceles triangle bottom specified by an angle $\theta_{o}$ to the vertical, as depicted in Fig. 1 (b). Each data point of $J_{o}$ or $J_{a}$ is obtained using 45 different initial conditions in steady state followed by 990,000 position-update cycles to ensure adequate sampling and to obtain error bars representing the standard deviation of each measured quantity.

\section{RESULTS AND DISCUSSIONS}

Below, we first plot the normalized hopper flow rates $J_{a} / J_{o}$ as a function of the total number of particles in the system $N$. We then show the flow rate enhancement is caused by the particle concentration mechanism below the obstacle by measuring the constituent variables, the local area packing fraction $\phi_{l}$ and the averaged particle velocity $v_{y}$ along the $y$ direction, of $J_{a} / J_{o}$. After that we investigate the importance of the particle concentration mechanism within the void below the obstacle in-between $y=[0, H]$ by artificially making the horizontal driving strength $\alpha_{x}$ anisotropic in this region, which mobilizes particles in the void space and increases the local area packing fraction $\phi_{l}$ near the hopper exit. We show that this strategy is actually a way of utilizing the concentration mechanism by manipulating particles below the obstacle. We also use an obstacle with a hollow duct of variable width $W_{d}$ to examine the sensitivity of the particle concentration mechanism. Finally, we explore the waiting room effect on the flow rate enhancement by using a semicircular obstacle with a triangular half of adjustable $\theta_{o}$, changing the vertical speed-up rate $r_{s}$, or varying the exit angle of the hopper $\theta_{2}$.

\section{A. The effect of the total number of particles in the system}

To test the effect of the total number of particles in the hopper $N$ on the flow rate, we tried ten different system sizes between $N=8$ and 2048 and measured the corresponding $J_{a} / J_{o}$. The results with $\alpha_{x}=1.0, \alpha_{y}=0.333$, and $r_{s}=1.0$ are shown in Fig. 2(a1) for normalized $J_{a} / J_{o}$ and Fig. 2(a2) for unnormalized $J_{a}$, respectively. Initially, $J_{a}$ increases rapidly with $N$. When $N$ is smaller than about 300 , the obstacle only slows down the normalized flow rate monotonically with increasing $N$ and diminishing $H$. However, when $N \geq 342, J_{a} / J_{o}$ becomes greater than 1.0, reflecting that $H$ exceeds a characteristic value, and exhibits a local peak. The enhanced flow rate can be achieved only when the obstacle is placed within an $H$ range that shrinks with increasing $N$ and finally saturates as $N$ approaches about 428 , the minimum number of particles required to reproduce the results at the large system size limit. A snapshot where a flow rate peak happens with $N=428$ is shown in Fig. 2(b). The outcome of the system size dependence test suggests that the flow rate enhancement is limited to a system of a sizable number of particles. As we reveal in the following sections, as particles move through the two channels between the obstacle and the hopper walls their trajectories concentrate and a slightly sparser but faster particle flow is formed and discharged at the hopper exit, as compared to the case without the obstacle. This produces a larger than unity $J_{a} / J_{o}$. A hopper containing too few particles cannot sustain the associated concentration mechanism crucial for the flow rate peak.

\section{B. The concentration mechanism causing the flow rate peaking phenomenon in the Tetris-like model}

To understand the flow rate peaking phenomenon, we decompose the flow rate into $J_{a}=\phi_{l} v_{y} w$ in terms of the local area packing fraction $\phi_{l}$, the width-averaged particle velocity $v_{y}$ (in the $y$ direction), and the passage horizontal width $w$ at vertical location $y_{c}$ where $v_{y}$ is measured. Specifically, we define $\phi_{l}\left(y_{c}\right)$ at $y=y_{c}$ as the ratio of the total area of particles, whose centers reside within the space confined by $y=y_{c}+\Delta_{y} / 2, y=y_{c}-\Delta_{y} / 2$, and the two hopper walls, to the available area of the con- 

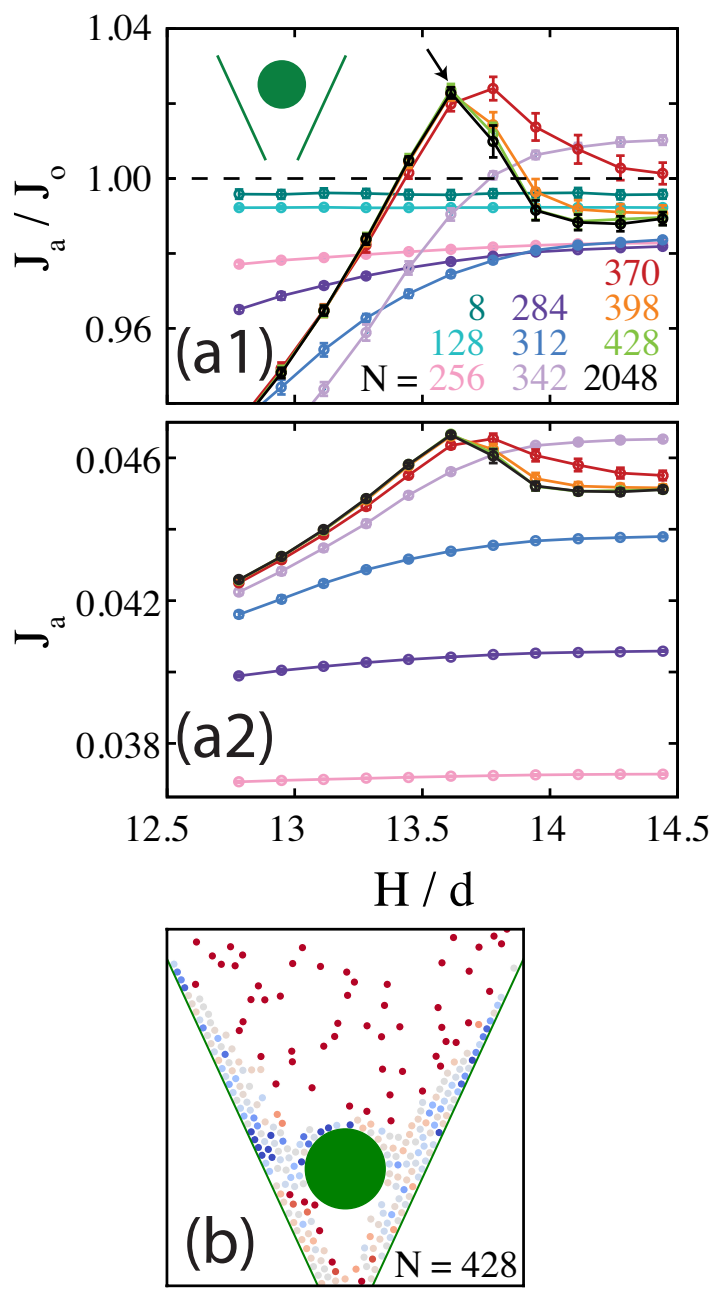

FIG. 2. (Color online) (a1) Normalized hopper flow rates $J_{a} / J_{o}$ measured at the exit of a hopper with $\theta_{2}=\theta_{1}$ and containing a round obstacle. The total number of particles in the system $N=8$ (dark green), 128 (cyan), 256 (pink), 284 (dark purple), 312 (navy), 342 (purple), 370 (red), 398 (orange), 428 (green), and 2048 (black). The driving strengths $\alpha_{x}=1.0, \alpha_{y}=0.333$, and the speed-up rate $r_{s}=1.0$. (a2) Same plot as (a1) except unnormalized hopper flow rates $J_{a}$ are shown. The curves of $N=8$ and 128 are not included because their $J_{a}$ values are outside the visible vertical range. (b) A snapshot focusing on the lower half of the hopper with $N=428$ where an obstacle is placed as indicated by the arrow in (a). Particles are colored by the blue-red scheme in Fig. 11.a).

fined space. Similarly, we define $v_{y}\left(y_{c}\right)=\Delta_{y} / \bar{t}$ at $y=y_{c}$, where $\bar{t}$ is the average position-update cycles required for a particle travels from $y=y_{c}+\Delta_{y} / 2$ to $y=y_{c}-\Delta_{y} / 2$. We chose a constant $\Delta_{y}=0.03 L \approx 2.49 d$ throughout this study. For a given system setup, we randomly choose 10 trials out of the 45 initial conditions to obtain the means and error bars of $\phi_{l}$ and $v_{y}$. Superscripted with a capital E, the calculated hopper flow rates at the exit with or without the obstacle may be expressed as $J_{a}^{E}=\phi_{l}^{E} v_{y}^{E} w^{E}$ or $J_{o}^{E}=\phi_{l o}^{E} v_{y o}^{E} w^{E}$, respectively. The local values of $\phi_{l}^{E}$,
$v_{y}^{E}, \phi_{l o}^{E}$, and $v_{y o}^{E}$ are measured at $y_{c}^{E}=0.015 L \approx 1.245 d$, only slightly above the hopper exit at $y=0$ for an accurate estimation, and $w^{E}$ is the corresponding orifice width at $y_{c}^{E}$. An extra subscript letter $o$ is added to distinguish the values measured without an obstacle. Putting everything together, we have the normalized flow rate $J_{a}^{E} / J_{o}^{E}=\left(\phi_{l}^{E} / \phi_{l o}^{E}\right)\left(v_{y}^{E} / v_{y o}^{E}\right)$ in terms of the product of the normalized local area packing fraction $\phi_{l}^{E} / \phi_{l o}^{E}$ and the normalized averaged particle velocity $v_{y}^{E} / v_{y o}^{E}$ at the hopper exit.

In the lower box of Fig. 3(a), we plot $J_{a} / J_{o}$ with $\alpha_{y}=0.333$ and $N=2048$, copied from Fig. 2(a1), together with its corresponding measured constituent variables $\phi_{l}^{E} / \phi_{l o}^{E}$ and $v_{y}^{E} / v_{y o}^{E}$ that give the calculated $J_{a}^{E} / J_{o}^{E}$. The calculated $J_{a}^{E} / J_{o}^{E}$ using $\phi_{l}^{E} / \phi_{l o}^{E}$ and $v_{y}^{E} / v_{y o}^{E}$ are in close agreement to the measured $J_{a} / J_{o}$, supporting our approach of variable decomposition and its estimation. As the obstacle is placed at a higher place above the hopper exit with increasing $H / d$, the two channels between the obstacle and the hopper walls become wider and allow more particles to move through. As a result, this creates a monotonically increasing $\phi_{l}^{E} / \phi_{l o}^{E}$ at the hopper exit. Correspondingly, a higher packing fraction comes with higher particle clogging probability, which reduces particle velocity. Therefore, we simultaneously observe a monotonically decreasing $v_{y}^{E} / v_{y o}^{E}$ at the hopper exit. Placing the obstacle too close to the hopper exit blocks too many particles, yielding in a low $\phi_{l}^{E} / \phi_{l o}^{E}$. On the other hand, placing the obstacle too far from the hopper exit results in $v_{y}^{E} / v_{y o}^{E}<1$ due to a high clogging probability from too many particles being discharged out of the hopper. Only an optimally placed obstacle blocks just enough particles so that $\phi_{l}^{E} / \phi_{l o}^{E}$ is slightly lower than 1 while also allowing particles to be discharged faster at the hopper exit with $v_{y}^{E} / v_{y o}^{E}>1$. This creates an enhanced flow rate, where a characteristic transition happens when $\phi_{l}^{E}>\phi_{o}^{c} \approx 0.55$, as discussed in the appendix Sec. V (variation of $\phi_{l}^{E}$ is shown in Fig. 3 (b) below). The characteristic transition experiences a positive feedback from the shrinking geometry of the hopper, which raises $\phi_{l}^{E}$ with decreasing $v_{y}^{E}$ while particles move through the hopper exit. Reciprocally, a slower $v_{y}^{E}$ increases $\phi_{l}^{E}$, which helps maintain the condition of $\phi_{l}^{E}>\phi_{o}^{c}$ until particles are discharged from the hopper. In the upper box of Fig. 3(a), we plot normalized bottleneck velocity $v_{y}^{B N} / v_{y o}^{E}$, measured at $y_{c}=H-0.015 L$, which exhibits a plateau before the flow rate peak and decreases again afterwards. The plateau is a signal that upstream particles at the bottleneck start to sense the downstream characteristic transition.

In Fig. 3(b), we plot the local area packing fraction $\phi_{l}(y)$ under the obstacle in-between $y=[0, H]$ (see the shaded zone in the inset) of the three selected cases, labeled in Fig. 3(a). We chose the zone in this way because $\phi_{l}$ exhibits the most significant variation in this region, and its pattern of variation depends on whether a system exhibits an enhanced flow rate or not, as reported in our 

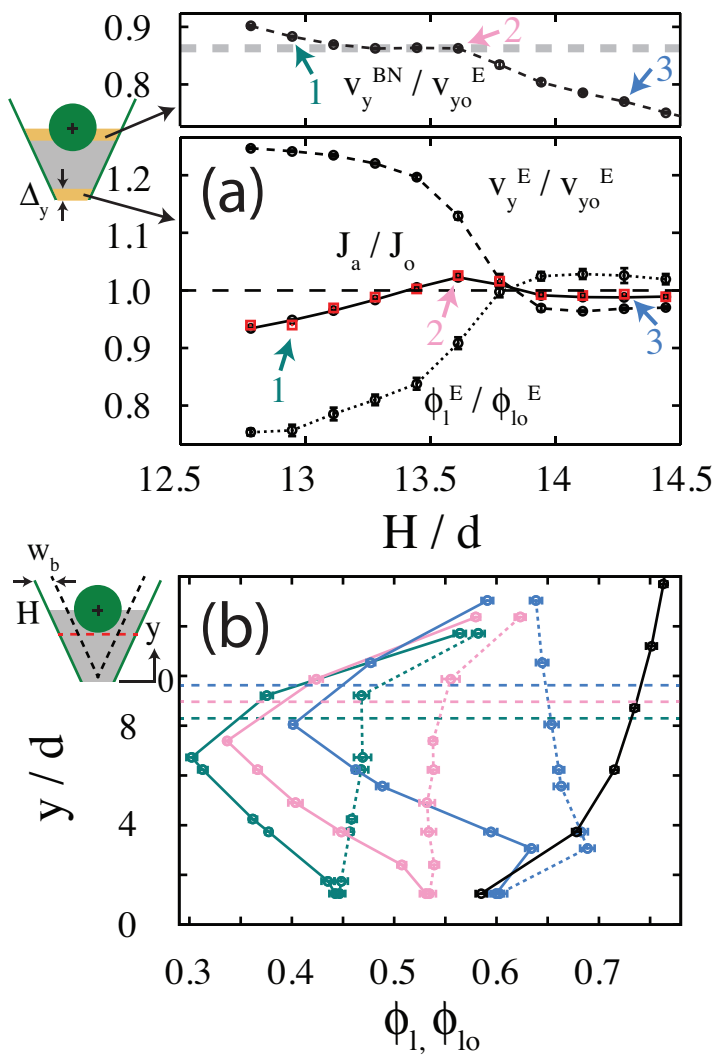

FIG. 3. (Color online) (a) The lower box shows a plot of normalized local area packing fraction $\phi_{l}^{E} / \phi_{l o}^{E}$ (dotted line), normalized averaged particle velocity $v_{y}^{E} / v_{y o}^{E}$ (dashed line), measured at $y_{c}=0.015 L \approx 1.245 d$ (lower yellow region in the inset). The calculated normalized flow rate using the product $J_{a}^{E} / J_{o}^{E}=\left(\phi_{l}^{E} / \phi_{l o}^{E}\right)\left(v_{y}^{E} / v_{y o}^{E}\right)$ is also marked (red squares). The corresponding normalized hopper flow rate $J_{a} / J_{o}$ (solid line) is copied from Fig. 2(a1) for reference. The upper box shows a plot of $v_{y}^{B N} / v_{y o}^{E}$, measured at bottleneck $y_{c}=H-0.015 L$ (upper yellow region in the inset). The dashed line indicates its plateau value. The driving strengths $\alpha_{x}=1.0, \alpha_{y}=0.333$, the speed-up rate $r_{s}=1.0$, and $N=2048$. (b) Averaged local area packing fraction $\phi_{l}$ in the shaded zone in-between $y=[0, H]$ for the three selected cases labeled in (a), where the obstacle is placed at 1 (dark green), 2 (pink), and 3 (navy). The dotted lines are $\phi_{l}$ next to the hopper wall within a stripe of width $w_{b} \approx 3.669 d$, limited by the black dashed lines marked on the inset. The horizontal dashed lines indicate the lowest $y$ positions of the obstacle. The black line is the corresponding averaged local area packing fraction $\phi_{l o}$ while the hopper contains no obstacle.

previous study [14. In all cases, $\phi_{l}$ first decreases to a local minimum somewhere below the obstacle due to the available void therein, after which $\phi_{l}$ increases because of the concentration of particles on their way towards the hopper exit. In the case after the flow rate peak (labeled as 3 in Fig. 3(a)), $\phi_{l}$ decreases again to facilitate the discharging of extremely densely-packed particles with $\phi_{l}>0.6$ near the hopper exit. To observe the true variation of $\phi_{l}$ without the interruption of the void below the obstacle, we also plot $\phi_{l}$ next to the hopper wall within a stripe of width $w_{b} \approx 3.669 d$, which is the narrowest width between the obstacle and the hopper wall when the obstacle is placed at the lowest position of the three selected cases. Both before and at the flow rate peak, $\phi_{l}$ next to the hopper wall still exhibits some decrease immediately after particles pass the narrowest channel segments, which implies particle acceleration. Afterwards, $\phi_{l}$ stays approximately constant until reaching the hopper exit. The fact that $\phi_{l}$ can maintain a constant value is not trivial and is caused by the shrinking geometry of the hopper exit generating a concentrating effect, which goes away as soon as we reduce the exit angle $\theta_{2}$, as shown later in Fig. 13(b). In contrast, $\phi_{l o}$ exhibits a monotonic decrease below the obstacle. By comparing $\phi_{l}$ with $\phi_{l o}$, we can see that introducing an obstacle into the hopper changes the discharging mechanism. When the hopper contains an obstacle near its exit, the two concentrated particle flows merge near the hopper exit before discharge. When the hopper contains no obstacle, on the other hand, a dense group of particles with $\phi_{l o}>\phi_{o}^{c}$ above the hopper exit has to dismantle first before exiting the hopper, as suggested by the progressively decreasing $\phi_{l o}$ towards the hopper exit.

Based on Fig. 3. our explanation of the flow rate enhancement by the concentration mechanism goes as follows. Before the peak, $\phi_{l}^{E}<\phi_{o}^{c}$, and the position of the obstacle controls the bottleneck flux $J_{a}^{B N}$ arriving at hopper exit. The exit adjusts its $\phi_{l}^{E}$ in order to maintain the steady state $J_{a}^{E}$, that is, to maintain the flux balance $J_{a}^{B N}=J_{a}^{E}$. In this regime, what happens at the hopper exit does not affect the flux $J_{a}^{B N}$. A representative snapshot is shown in Fig. 4(a1), where we can see that there are few particles at the exit because $\phi_{l}^{E}$ is low. On the other hand, at or after the peak, $\phi_{l}^{E} \geq \phi_{o}^{c}$, as shown in Fig. 4(a2) and (a3). The particles with $\phi_{l}^{E} \geq \phi_{o}^{c}$ at the hopper exit work together like a plug blocking the flow, and therefore $J_{a}^{B N}>J_{a}^{E}$. In this situation, the steady state with no coupling between the downstream exit and the upstream bottleneck regions is not possible. If we further place the obstacle at a higher position, the area packing fraction in the entire region between the obstacle and the exit will build up, until the particles block the flow through the bottleneck created by the obstacle, as shown in Fig. 44(a4). The plateau of $v_{y}^{B N}$ is an initial response to the downstream-upstream (exitbottleneck) coupling. Then $v_{y}^{B N}$ drops after the flow rate peak, similar to a transition from the free-flow regime to the congested-flow regime where the waiting room effect occurs 4].

Finally, a hopper discharging relatively strongly-driven particles with a larger $\alpha_{y}=0.439$ exhibits no flow rate enhancement. This is because the converging geometry of the hopper cannot effectively concentrate particles with a high $v_{y}^{E} / v_{y o}^{E}>1$ while discharging them and $\phi_{l}^{E}$ never becomes higher than $\phi_{o}^{c}$ to make a flow rate peak. This is inspected similarly in Fig. 6(a, b1) in the next section, where we also propose an artificial merging strategy by 




FIG. 4. (Color online) (a1-a3) Representative snapshots of the system, focusing on the zone below the obstacle, placed at 1, 2, and 3 as labeled in Fig. 3(a). (a4) Another snapshot where the obstacle is placed much further away from the exit of the hopper with $H / d \approx 21.414$. Particles are colored by the blue-red scheme in Fig. 1.a), and their properties at the hopper exit are measured within the yellow region of height $\Delta_{y}=0.03 L$.

utilizing the concentration mechanism to create a manmade flow rate enhancement peak.

\section{The importance of particle concentration mechanism within the space below the obstacle}

To further validate the effect of particle concentration mechanism within the domain below the obstacle on the flow rate, we artificially compel particles to merge horizontally on their way toward the hopper exit when they have moved to the shaded zone marked in the inset of Fig. 3(b). We achieve this by introducing an anisotropic driving strength $\alpha_{x}=m \geq 1.0$ for particles in the domain in-between $y=[0, H]$ that encourages them to attempt moving horizontally towards the centerline of the hopper. On the other hand, if particles in the same domain attempt to move horizontally away from the centerline of the hopper, $\alpha_{x}=1.0$ as usual. In summary, $\alpha_{x}=\left\{\begin{aligned} m, & \text { towards the centerline } \\ 1, & \text { otherwise }\end{aligned}\right.$. This artificial merging strategy presumably improves concentrating efficiency beneath the obstacle by guiding particles to where more space is available and reducing their probability of hitting the hopper walls. We find that applying this strategy can generate a higher flow rate peak of weakly-driven particles or can even achieve flow rate enhancement in a relatively strongly-driven system that originally exhibits no such phenomenon, as shown in Fig. $5(\mathrm{a} 1)$ and (a2), respectively.

In Fig. 5(a1), we plot the normalized flow rate $J_{a} / J_{o}$ as a function of $H / d$ using the same $\alpha_{y}=0.333$. When $m=1.0$, the system exhibits the same flow rate peak without the artificial merging strategy, as shown in Fig.
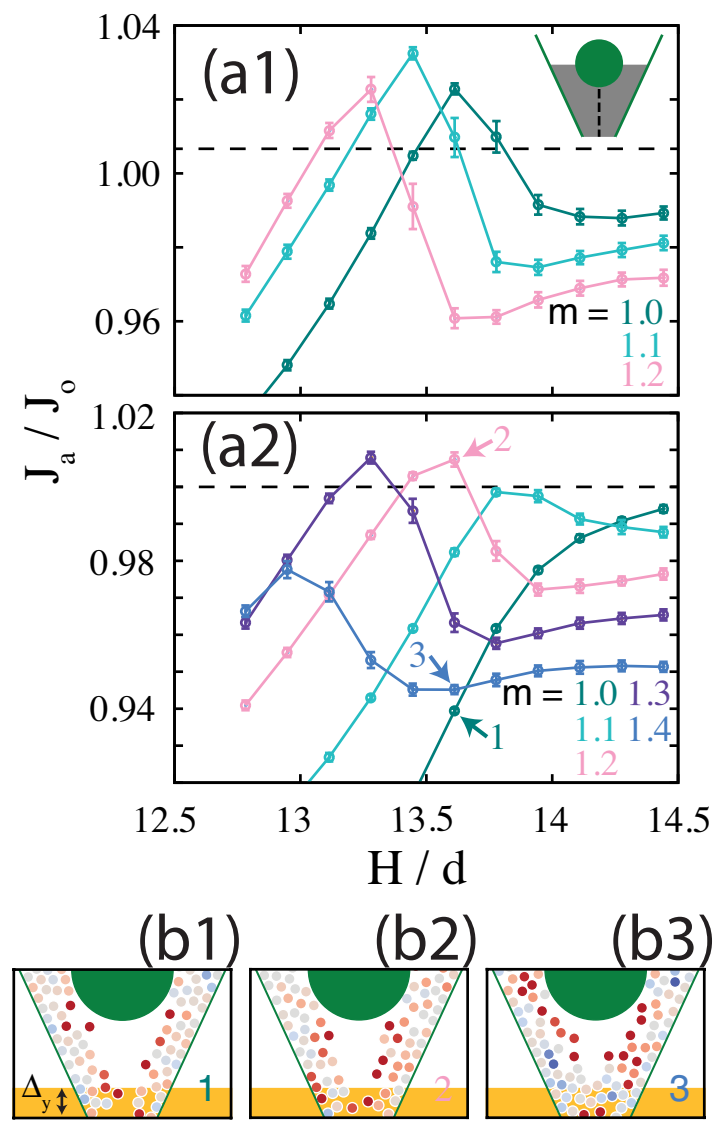

FIG. 5. (Color online) (a1-a2) Normalized hopper flow rates $J_{a} / J_{o}$ measured at the exit of a hopper with $\theta_{2}=\theta_{1}$, containing a round obstacle and $N=2048$. The driving strength $\alpha_{x}=m$ is 1.0 (dark green), 1.1 (cyan), 1.2 (pink), 1.3 (dark purple), and 1.4 (navy) if particles in the shaded zone inbetween $y=[0, H]$ move horizontally closer to the centerline (vertical dashed line) of the hopper, as shown by the inset. Otherwise, $\alpha_{x}=1.0$. The vertical driving strength $\alpha_{y}=0.333$ in (a1) and 0.439 in (a2), respectively. The speedup rate $r_{s}=1.0$. (b1-b3) Representative snapshots of the system, similar to Fig. 4 but representing the three cases indicated by arrows in (a2).

2(a1) and Fig. 3(a). We can maximize the peak value by using an optimal $m=1.1$. In Fig. 5 (a2), we plot $J_{a} / J_{o}$ with an amplified $\alpha_{y}=0.439$ and the system exhibits no flow rate peak when $m=1.0$ within the same range of $H / d$. However, we can see that $J_{a} / J_{o}$ exhibits a local peak shifting to the left as $m$ is increased. The peak value of $J_{a} / J_{o}$ becomes larger than unity as $m$ falls between 1.2 and 1.3. We argue that using $m=1.2$ decreases the probability of particles below the obstacle hitting the hopper walls, which allows them to merge more smoothly on their way towards the hopper exit in the available space near the centerline, promoting a higher maximum flow rate as they accumulate. Further increasing the value of $m$ to 1.4 lowers the enhanced flow rate because particles begin to experience more failed moves near the centerline of the hopper due to more frequent 
interparticle interference and can even clog at its exit, yielding less effective concentration mechanism. We first verify the above argument visually using the representative snapshots of the selected cases shown in Fig. 5(b1) - (b3), where particles at the hopper exit experience the characteristic transition from $\phi_{l}^{E}<\phi_{o}^{c}$ to $\phi_{l}^{E}>\phi_{o}^{c}$, a similar scenario as in Fig. 4. Next, we put our arguments under quantitative investigation and show the results in Fig. 6 and Fig. 7.

The artificial merging strategy can be interpreted as a way of utilizing the concentration mechanism in Sec. IIIB to create a flow rate enhancement peak. To show this, we plot $J_{a} / J_{o}$ with $\alpha_{y}=0.439$ and $m=1.0$, copied from Fig. 5(a2), together with its measured decomposed variables $\phi_{l}^{E} / \phi_{l o}^{E}$ and $v_{y}^{E} / v_{y o}^{E}$, and the calculated $J_{a}^{E} / J_{o}^{E}$ in the lower box of Fig. 6(a). As in Fig. 3(a), $\phi_{l}^{E} / \phi_{l o}^{E}$ and $v_{y}^{E} / v_{y o}^{E}$ at the hopper exit monotonically increases and decreases, respectively, with increasing $H / d$ as the obstacle is placed further away from the hopper exit. However, $\phi_{l}^{E}$ is never greater than $\phi_{o}^{c}$ while maintaining a high $v_{y}^{E}$ to create the characteristic transition shown in Fig. 3(a), and therefore we do not observe an enhanced flow rate. Now, we switch on the artificial merging strategy by increasing $m=1.0$ to $m=1.2$ and 1.4, labeled as cases 1 , 2, and 3 in the dashed box in Fig. 6(a) (corresponding to identically labeled cases in Fig. 5(a2)). Together, we mark how $J_{a}^{E} / J_{o}^{E}$ and the associated $\phi_{l}^{E} / \phi_{l o}^{E}$ and $v_{y}^{E} / v_{y o}^{E}$ change with $m$, indicated by the yellow arrows in the figure. Using $m=1.2$ successfully increases $\phi_{l}^{E} / \phi_{l o}^{E}$ while $v_{y}^{E} / v_{y o}^{E}$ is only mildly reduced at the hopper exit. As a result, we observe $J_{a}^{E} / J_{o}^{E}>1$. A larger $m=1.4$ further increases $\phi_{l}^{E} / \phi_{l o}^{E}$ but simultaneously $v_{y}^{E} / v_{y o}^{E}$ becomes too small to make $J_{a}^{E} / J_{o}^{E}>1$. Again, the calculated $J_{a}^{E} / J_{o}^{E}$ agrees with the measured $J_{a} / J_{o}$ well in all studied cases. In the upper box of Fig. 6(a), we plot $v_{y}^{B N} / v_{y o}^{E}$, which exhibits a gentle local peak. The peak indicates a weak downstream-upstream coupling. Nevertheless, the overall effect of the concentration mechanism on relatively strongly-driven particles is not significant enough to create a flow rate enhancement peak.

To learn more about the variation of $\phi_{l}$ under the obstacle placed at different $H / d$ without the artificial merging strategy $(m=1.0)$, we select three $H / d$, labeled as 1-, 1, and 1+ in Fig. 6(a), and plot their $\phi_{l}$ in Fig. 6(b1). In all the cases, $\phi_{l}$ first decreases until somewhere below the obstacle and then increases until the hopper exit. Plotting $\phi_{l}$ next to the hopper wall reveals that the densest part of the local area packing fraction in this region decreases monotonically towards the hopper exit, which indicates that the converging geometry of the hopper does not effectively help to collect particles on their way out. Its value is even smaller than when the hopper contains no obstacle. While the particles move fast towards the hopper exit because of a large $\alpha_{y}$, particles with a decaying $\phi_{l}$ passing the obstacle travel even faster so long as the concentrating capability of the hopper fails to keep $\phi_{l}$ next to the hopper wall constant, as is the case


FIG. 6. (Color online) (a) The same plot as Fig. 3(a) with $N=2048$ and $\alpha_{y}=0.439$ but different $\alpha_{x}=m$ to clarify the artificial merging strategy. Three selected cases with $m=1.0$ at different $H / d$ are labeled as 1 - (dark purple), 1 (dark green), and $1+$ (red) while another two cases with $m=1.2$ and 1.4 at $H / d$ of case $1(=13.612)$ are labeled as 2 (pink) and 3 (navy), respectively. The data for cases 1,2 , and 3 in the dashed box are $v_{y}^{E} / v_{y o}^{E}, J_{a}^{E} / J_{o}^{E}$ on top of duplicate $J_{a} / J_{o}$, and $\phi_{l}^{E} / \phi_{l o}^{E}$ from left to right (shifted horizontally for better visibility). The orange arrows indicate the direction of increasing $m$. The corresponding normalized hopper flow rate $J_{a} / J_{o}$ with $m=1.0$ (solid line) and $m=1.2$ and 1.4 (circles) are copied from Fig. 5(a2) for reference. (b1) The same plot as Fig. 3(b), except for cases 1-, 1, and 1+. (b2) The same plot as (b1), except for cases 1, 2, and 3 .

in Fig. 3(b), which reciprocally further reduces $\phi_{l}$ next to the hopper wall below the obstacle. Therefore, $\phi_{l}^{E} / \phi_{l o}^{E}$ cannot reach a high enough value with a still fast $v_{y}^{E} / v_{y o}^{E}$ to create a flow rate enhancement.

In Fig. 6(b2), we plot $\phi_{l}$ under the obstacle with a fixed $H / d=13.612$ but different strengths of the artificial merging strategy with $m=1.0,1.2$, and 1.4, labeled as 1, 2, and 3 in Fig. 5(a2) or Fig. 6(a). The value 
of $\phi_{l}$ again decreases monotonically from the narrowest points between the obstacle and the two hopper walls to somewhere below the obstacle. Compared with particles subject to an isotropic horizontal $\alpha_{x}=m=1, \phi_{l}$ of particles with $m=1.2$ is smaller in the vicinity below the obstacle but soon grows above all the way towards the exit. The rise of $\phi_{l}$ is more effective than the slight drop of $v_{y}$ to lead to the flow rate enhancement. When we overdo the artificial merging strategy with an $m=1.4$, $\phi_{l}$ grows much more severely towards the exit and even exhibits a sudden drop before the exit, destroying the flow rate enhancement, similar to the after-peak case 3 presented in Fig. 3(b). In addition, we plot $\phi_{l}$ next to the hopper wall with the dotted lines, whose value with $m=1.0$ decreases monotonically, as we have seen previously. In contrast, both the cases with $m=1.2$ and 1.4 decrease only initially but then increase, but a sudden drop close to the exit is detected when overdoing the artificial merging strategy with $m=1.4$. We notice that the nonlinear variation of $\phi_{l}$ near the hopper exit with increasing $m$ closely resembles the trend found before, at, and after an enhanced flow rate peak by moving the obstacle up in a hopper discharging isotropic particles, as shown in Fig. 3(b).

Focusing on the particle data with an anisotropic $\alpha_{x}$ collected in the shaded space in the inset of Fig. 6(b1), we go one step further to quantitatively examine the complementary cumulative distribution function $P(n \geq \nu)$ with respect to $m$, which gives the probability of randomly finding an $n$ no smaller than $\nu$ [17. The value of $n$ represents particles that successfully or unsuccessfully update their positions $n^{s}$ or $\left|n^{f}\right|$ times. We perform the calculation using 990,000 position-update cycles after a system forgets its initial state. We also calculate the corresponding $N^{t} / N_{0}^{t}$, where $N^{t}$ is the total number of successful-type particles $N^{s}$ or failed-type particles $N^{f}$ counted while building $P\left(n^{s} \geq \nu\right)$ or $P\left(\left|n^{f}\right| \geq \nu\right)$, and $N_{0}^{t}$ is the same quantity with $m=1.0$ used for normalization. The resulting $P(n \geq \nu)$ of the three selected cases with the same $H / d$, labeled as 1,2 , and 3 in Fig. 5(a2) or Fig. 6(a), are shown in Fig. 7(a). As $m$ increases from 1.0 to 1.2 , the range of $\nu$ with $P\left(\left|n^{f}\right| \geq \nu\right)$ between 0.1 and 1.0 barely expands, meaning that about $90 \%$ of the motionless particles do not experience a higher failure rate of updating their positions. On the other hand, $P\left(n^{s} \geq \nu\right)$ shows an overall increase which leads to the improved flow rate. As $m$ increases again from 1.2 to 1.4, $P\left(n^{s} \geq \nu\right)$ shows no significant increase while $P\left(\left|n^{f}\right| \geq \nu\right)$ notably increases, resulting in the drop of the flow rate. The plot of $N^{t} / N_{0}^{t}$ against $m$ directly confirms this observation, as shown in Fig. 7(b). Compared with the case of $m=1.0$, the case of $m=1.2$ has its number of failed particles $N^{f}$ reduced by about $15 \%$ and number of successful particles $N^{s}$ increased by about $5 \%$. These numbers are also superior to those of the case with $m=1.4$, which have almost no improvement on $N^{s}$ but a dramatic increase in $N^{f}$ by almost $20 \%$.
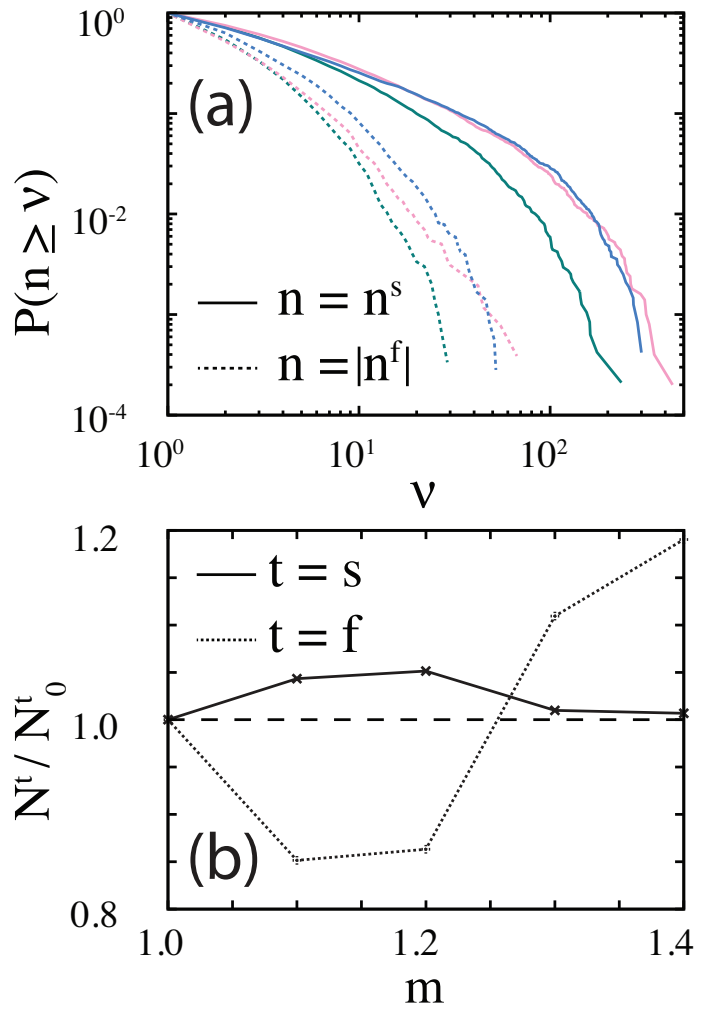

FIG. 7. (Color online) Representative (a) $P(n \geq \nu)$, using log-10 scales for both axes, and (b) $N^{t} / N_{0}^{t}$ in the shaded zone in-between $y=[0, H]$, as shown in the inset of Fig. 6.(b1), of the three selected cases, where a round obstacle is placed at $H / d=13.612$, indicated by arrows in Fig. 5(a2) with $m=1.0$ (dark green), 1.2 (pink), and 1.4 (navy).

\section{The sensitivity of particle concentration mechanism within the space below the obstacle}

To understand if simply filling the available space under the obstacle can also improve the flow rate, we introduce a different obstacle with a hollow duct of width $W_{d}>d$. The hollow duct offers a shortcut that allows particles to arrive at the available space below the obstacle faster, potentially leading to a more efficient particle concentration mechanism. The results are shown in Fig. 8. Counterintuitively, this approach has very limited effect on improving the flow rate, except when the duct width is very narrow with $W_{d}=1.5$. Much wider duct widths $\left(W_{d}>1.5\right)$ that allow more particles through can even have a negative influence on the enhanced flow rate. This approach probably hinders the original particle concentrating process due to particle exclusion upon collision. Additionally, particles passing through the uniform duct are subject to little concentrating assistance. Assigning an anisotropic $\alpha_{x}$ to particles below the obstacle, on the other hand, is a more effective strategy which improves the concentrating efficiency by directly reducing the collision probability between particles and 




FIG. 8. (Color online) Normalized hopper flow rates $J_{a} / J_{o}$ measured at the exit of a hopper with $\theta_{2}=\theta_{1}$, and containing a round obstacle with a hollow duct of width $W_{d}=0.0 d$ (dark green), 1.5d (cyan), 2.0d (pink), 2.5d (dark purple), $3.0 d$ (navy), and $4.0 d$ (purple). The total number of particles in the system $N=2048$, the driving strengths $\alpha_{x}=1.0$, $\alpha_{y}=0.333$, and the speed-up rate $r_{s}=1.0$.

the hopper.

\section{E. Investigating factors related to the waiting room effect}

The waiting room effect, wherein particles are first slowed down by the obstacle and then speed up due to the external driving force within the triangular-ish void space between the obstacle and the hopper exit, has been suggested to be responsible for the enhanced flow rate. Here we examine the factors related to the waiting room effect with the Tetris-like model by looking at its components one by one, namely the size of the void space, particle speed-up rate, and exit geometry of the hopper.

\section{The size of the waiting room}

To understand the effect of the triangular-ish void space on the enhanced flow rate, we replace the round obstacle by one composed of an identical upper semicircle and a lower triangle with an area $A_{t}$ and an angle $\theta_{o}$ measured from the vertical, as shown in Fig. 9. We keep the shape of the upper half of the obstacle unchanged so that we can focus on the size of the waiting room, which is controlled by the lower half of the obstacle. When $\theta_{o}=\pi / 2, A_{t}=0$. We define the maximum waiting room area $A_{w}^{m}$ as the space circumscribed by the lower boundary of the semicircle and the two lines parallel to the hopper walls, as indicated by the dashed triangle in the insets of Fig. 9. The net waiting room area is found by deducting the area occupied by the lower triangle of the obstacle, $A_{w}=A_{w}^{m}-A_{t}$. It decreases with decreasing $\theta_{o}$ because of the associated increase of $A_{t}$. We tested seven



FIG. 9. (Color online) Normalized hopper flow rates $J_{a} / J_{o}$ measured at the exit of a hopper with $\theta_{2}=\theta_{1}=0.4325$, and containing an obstacle composed of a semicircle and an isosceles triangle with an area $A_{t}$ and an angle to the vertical $\theta_{o}=0.7850$ (dark green), 0.4325 (cyan), 0.40 (pumpkin), 0.38 (dark purple), 0.36 (navy), 0.34 (purple), and 0.32 (red). The total number of particles in the system $N=2048$, the driving strengths $\alpha_{x}=1.0, \alpha_{y}=0.333$, and the speed-up rate $r_{s}=$ 1.0. The insets are three representative system setups whose $J_{a} / J_{o}$ is at a peak value (denoted by the arrows), with the net waiting room area $A_{w}=A_{w}^{m}-A_{t}>0(\mathbf{A}),=0(\mathbf{B})$, and $<0(\mathbf{C})$, where $A_{w}^{m}$ is the maximum waiting room area (dashed triangles). The light green area in $(\mathbf{C})$ indicates the shrinking geometry from the obstacle to the hopper exit.

different values of $\theta_{o}$ between 0.7850 and 0.32 . When $\theta_{o}=0.7850$, the area of the composite obstacle can be fully encompassed by the round obstacle used before. We monitored the corresponding normalized flow rate $J_{a} / J_{o}$ and observed that a flow rate peak appears in all the tested cases. We can see that the value of the flow rate peak decreases slightly as $A_{w}$ changes from positive with $\theta_{o}=0.7850$ to zero with $\theta_{o}=\theta_{1}=0.4325$, showing that the waiting room size affects the enhanced flow rate but is not essential to it. Surprisingly, as we further decrease $\theta_{o}$ to about 0.36 the value of the flow rate peak increases and reaches an optimum with $A_{w}<0$, where the widths of the two channels between the obstacle and the hopper walls gradually shrink and particles must become more concentrated while flowing through them. Similar results with $A_{w}<0$ and $A_{w}>0$ have been reported experimentally and numerically using frictional particles in a quasi-2D hopper governed by Newtonian dynamics [6] however, here we test the case of $A_{w}=0$ and leave all other factors untouched. It is clear that eliminating the waiting room by reducing its area to zero does not completely annihilate the enhanced flow rate peak but only affects its magnitude.

Our test shows that we can create an enhanced flow 
rate even though the size of the waiting room is reduced to zero. To examine if the mechanism in Sec. IIIB can still explain the phenomenon in this setup, we consider the lower box of Fig. 10(a1). Here, we plot the measured $J_{a} / J_{o}$ again with the waiting room area $A_{w}=0$ and $\theta_{o}=0.4325$ (copied from Fig. 9), its measured $\phi_{l}^{E} / \phi_{l o}^{E}$ and $v_{y}^{E} / v_{y o}^{E}$, and the calculated $J_{a}^{E} / J_{o}^{E}$.

Our results show that $\phi_{l}^{E} / \phi_{l o}^{E}$ and $v_{y}^{E} / v_{y o}^{E}$ at the hopper exit follow identical patterns of monotonically increasing and decreasing, respectively, when the obstacle is placed further away from the hopper exit, as we have seen in Fig. 3(a). Similarly, the flow rate peaks where the characteristic transition in Fig. 3(a) happens. The results confirm the validity of the particle concentration mechanism. In the upper box of Fig. 10(a1), we plot $v_{y}^{B N} / v_{u o}^{E}$, which exhibits a similar plateau to the one in Fig. 3(a). We also plot the corresponding local area packing fraction $\phi_{l}$ under the obstacle in-between $y=[0, H]$ in Fig. 10(a2). The variation of $\phi_{l}$ becomes noticeably smaller but still visible after we eliminate the waiting room area in Fig. 3 (b). The visible variation of $\phi_{l}$ may suggest interparticle spatial fluctuations, even though the widths of the channels between the obstacle and the hopper walls stay constant. Similar spatial fluctuations in packing fraction have been reported both experimentally and numerically in a hopper or silo discharging frictional granular materials [18 21]. Before or after the flow rate peak, the value of $\phi_{l}$ decreases or increases monotonically from the narrowest points between the obstacle and the two hopper walls to somewhere above the lowest point of the obstacle. At the flow rate peak, the variation of $\phi_{l}$ is the smallest but also the most complicated. After the lowest point of the obstacle and immediately before the hopper exit, $\phi_{l}$ increases in all three cases due to the merging of the two channels and decreasing of the available space to the particles. In addition, by plotting the instantaneous particle $x$ and $y$ positions under the obstacle for the case of the maximum flow rate enhancement, we can see the related fluctuation of $\phi_{l}$ on a particle scale, as shown in Fig. 10(a3).

Likewise, in Fig. 10(b1), we plot $\phi_{l}$ with $A_{w}<0$ and $\theta_{o}=0.36$. The shrinking geometry from the obstacle to the hopper exit increases the degree of fluctuation of $\phi_{l}$ which also reflects on the particle $x$ and $y$ position scatter plot in Fig. 10(b2). These results of $\phi_{l}$ with $A_{w}=0$ and $A_{w}<0$ show that as long as $\phi_{l}$ can reach a value between 0.5 and 0.6 upon reaching the hopper exit, a flow rate enhancement can be achieved and the intermediate fluctuation of $\phi_{l}$ is not essential to the peaking phenomenon.

\section{The acceleration of particles within the waiting room}

The second factor associated with the waiting room effect is the acceleration of particles in the direction aligned with the external driving force such as gravity. This factor is expressed by the term $r_{s}^{n_{i}^{s}}$ in Eqn. 11 where a


FIG. 10. (Color online) a1) The same plot as Fig. 3(a), except the waiting room area $A_{w}=0$ and $\theta_{o}=0.4325$. (a2) $\phi_{l}$ in-between $y=[0, H]$ for cases labeled as 1 (dark green), 2 (pink), and 3 (navy) in (a1) and in the inset. The horizontal dashed lines indicate the lowest y positions of the obstacle. (a3) The corresponding instantaneous $x$ and $y$ position scatter plot of particles in the shaded zone with the same $y$ range for case 2 in (a2). The plot is obtained by overlapping 99 snapshots, each separated by 10,000 position-update cycles. (b1-b2) The same plots as (a2-a3), except $\theta_{o}=0.36$.

greater than unity $r_{s}$ is the speed-up rate that allows particle $i$, after successfully updating its position $n_{i}^{s}$ times, to move farther during the next position-update cycle. We test the concentration mechanism in Sec. IIIB with $r_{s}=1.01$ and show that it can still explain the flow rate enhancement even with particle acceleration, as seen in Fig. 11. Both $v_{y}^{B N} / v_{y o}^{E}$ and $J_{a} / J_{o}$ locally peak at the same value of $H / d$, similar to the transition from the 




FIG. 11. (Color online) The same plot as Fig. 3(a) with $\alpha_{x}=1.0, \alpha_{y}=0.333$, and $N=2048$, but a larger $r_{s}=1.01$, which allows a particle successively updating its position to move farther during the next position-update cycle.

free-flow regime to the congested-flow regime where the waiting room effect happens [4].

Including $r_{s}=1.01$, we also tested three other values of $r_{s}$ from 1.02 to 1.04 and compared the obtained normalized flow rate $J_{a} / J_{o}$ with that of $r_{s}=1.00$, the condition with no particle acceleration. The results are shown in the lower box of Fig. 12(a). We can see that both the peak value and the range of $H / d$ of the enhanced flow rate increase with a milder rise of $r_{s}=1.01$ and 1.02 . However, using an even higher particle speed-up rates towards to hopper exit $\left(r_{s}>1.02\right)$ has a negative impact on the flow rate peak value, as shown by the decreases in the two measured quantities. This decline may also be attributed to a higher collision frequency when more particles accelerate towards the hopper exit. In the upper box of Fig. 12 (a), we plot $v_{y}^{B N} / v_{y o}^{E}$, which plateaus with $r_{s}=1.00$ or peaks with $r_{s}>1$. Four representative snapshots with $r_{s}=1.03$ are shown in Fig. 12(b1) - (b4). The peaks of $v_{y}^{B N} / v_{y o}^{E}$ happen ahead of those of $J_{a} / J_{o}$ with increasing $r_{s}$, which indicates that upstream particles subject to stronger acceleration can detect downstream particles partially blocking the hopper exit earlier. Thus, the corresponding peak of $v_{y}^{B N} / v_{y o}^{E}$, signaling the downstream-upstream coupling, also occurs earlier, as shown in Fig. 12(b2).

\section{The geometry of the hopper exit}

Lastly, we assess whether shrinking the geometry of the void space from the obstacle to the hopper exit can effectively assist the particle concentration mechanism and the flow rate enhancement. We test this by varying the exit angle $\theta_{2}$ of a hopper with a round obstacle placed at a fixed position where a flow rate peak appears. Here, we chose the system with $N=2048, \alpha_{x}=1.0, \alpha_{y}=0.333$,
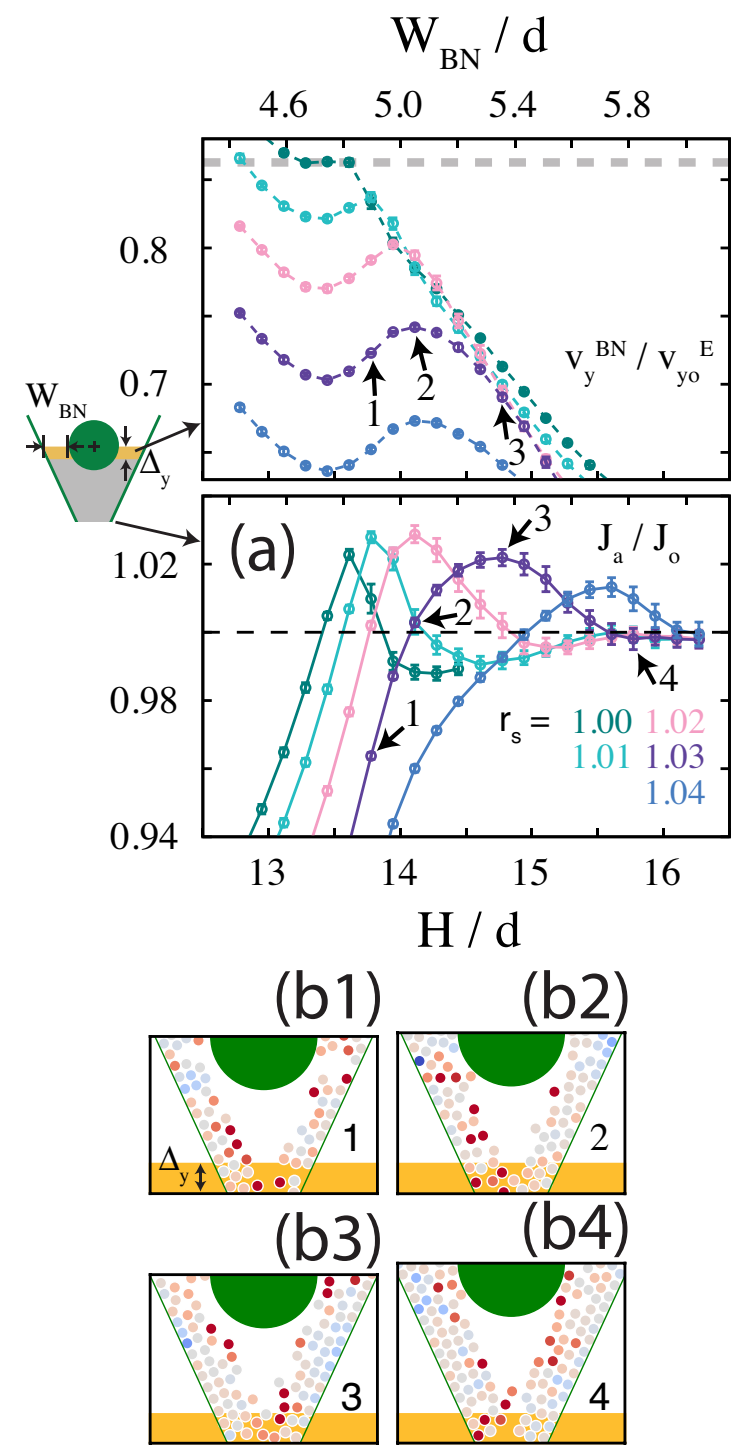

FIG. 12. (Color online) (a) The lower box shows a plot of normalized hopper flow rates $J_{a} / J_{o}$ measured at the exit of a hopper with $\theta_{2}=\theta_{1}$, containing a round obstacle. The upper box shows a plot of normalized averaged particle velocity $v_{y}^{B N} / v_{y o}^{E}$, measured at bottleneck $y_{c}=H-0.015 L$ (yellow region in the inset). The driving strengths $\alpha_{x}=1.0$ and $\alpha_{y}=0.333$, and $N=2048$. The speed-up rate $r_{s}=1.00$ (dark green), 1.01 (cyan), 1.02 (pink), 1.03 (dark purple), and 1.04 (navy). The lower and upper horizontal axes are normalized obstacle position $H / d$ and normalized bottleneck width $W_{B N} / d$, respectively. (b1-b4) Representative snapshots of the system, similar to Fig. 4, illustrating the four cases indicated by arrows in (a).

and $r_{s}=1.0$ so that $J_{a} / J_{o}$ peaks at $H / d=13.612$, as previously shown by the black line in Fig. 2(a1). The results are shown in Fig. 13(a). We find that the value of the flow rate peak drops immediately as soon as the hopper walls become uneven with an opening hopper exit, represented by $\theta_{2} / \theta_{1}<1.0$, where $\theta_{1}$ is the fixed hopper angle. These results prove that the shrinking geometry 

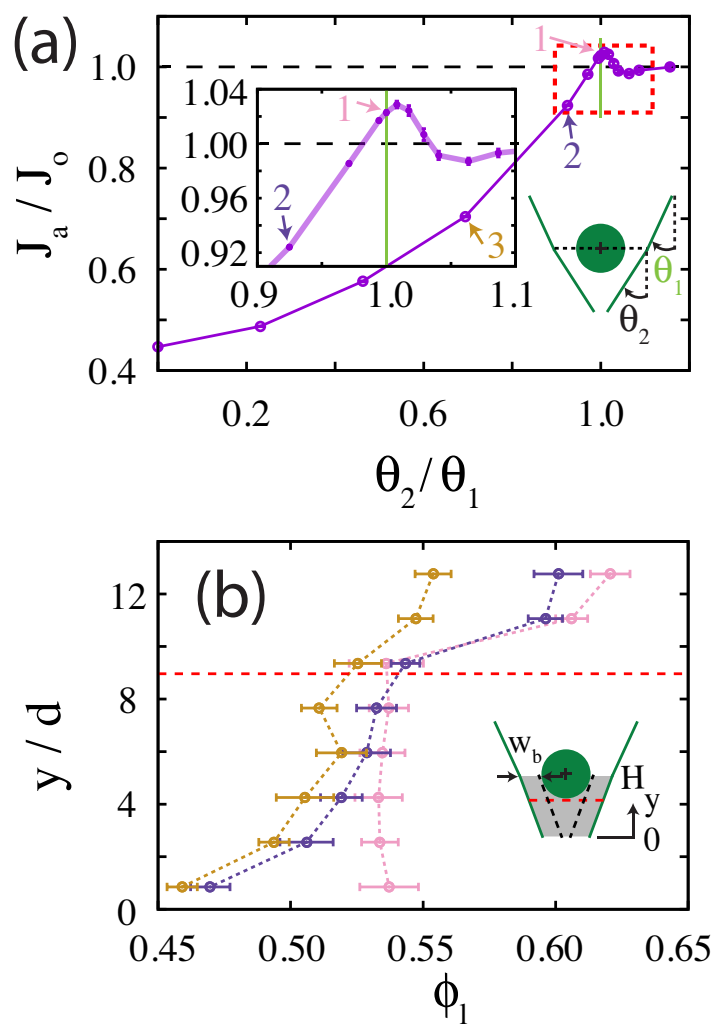

FIG. 13. (Color online) (a) Normalized hopper flow rate $J_{a} / J_{o}$ measured at the exit of a hopper with its exit angle $\theta_{2}$ variable, containing a round obstacle placed at $H / d=$ 13.612 , and $N=2048$. The driving strengths $\alpha_{x}=1.0, \alpha_{y}=$ 0.333 , and the speed-up rate $r_{s}=1.0$. The green vertical line indicates where $\theta_{2}$ equals the hopper angle $\theta_{1}=0.4325$ and the two hopper walls are flat. The inset is a zoom-in of the red-dashed region. (b) Averaged local area packing fraction $\phi_{l}$ in the shaded zone in-between $y=[0, H]$ and next to the hopper wall within a stripe of width $w_{b} \approx 3.669 d$, as shown in the inset. The three selected cases correspond to those identified in the inset of $(\mathrm{a}): \theta_{2} / \theta_{1}=1.0$ (pink), $\approx 0.925$ (dark purple), and $\approx 0.694$ (brown). The horizontal dashed line indicates the lowest $y$ positions of the obstacle.

is crucial, a conclusion that can also be inferred from the particle concentration mechanism in Sec. IIIB. In contrast, if $\theta_{2}$ is varied in the opposite direction to become uneven with a closing hopper exit, represented by $\theta_{2} / \theta_{1}>1.0$, there exists a very narrow range of $\theta_{2} / \theta_{1}$ between 1.0 and 1.029 that further enhances the peak of $J_{a} / J_{o}$ from the flat hopper design. Beyond that, no enhanced flow rate, $J_{a} / J_{o} \geq 1$, is observed due to the high clogging probability produced by the increasingly narrow hopper exit. In Fig. 13(b), we plot $\phi_{l}$, below the obstacle and next to the hopper wall within a stripe of width $w_{b}$, of three selected $\theta_{2} / \theta_{1} \leq 1$ labeled in Fig. 13(a). The results show that if $\theta_{2} / \theta_{1}<1, \phi_{l}$ in this region cannot maintain a constant value and decreases monotonically, again confirming the necessity of the shrinking geometry of the hopper exit for creating a flow rate peak.
In section IIIC, we created an enhanced flow rate peak in a system with $\alpha_{y}=0.439$ that originally exhibits no such phenomenon using an anisotropic $\alpha_{x}$ below the obstacle. Although effective, the strategy could be criticized for being artificial and difficult to reproduce and verify experimentally. Here we create a flow rate peak again in the same system by narrowing the hopper below the obstacle, a setup which is more practical. We show the enhanced $J_{a} / J_{o}$ in Fig. $14(\mathrm{a})$, where the value of $J_{a} / J_{o}$ increases from 0.94 to 1.01 , or by about $7 \%$, when $\theta_{2} / \theta_{1}$ increases from 1.0 to 1.058 . It should be noted that $J_{o}$ used for normalization decreases with increasing $\theta_{2}$ because the hopper opening becomes narrower. Therefore, this method is inherently at a disadvantage as it does not enhance the unnormalized flow rate $J_{a}$. In Fig. 14(b), we plot $\phi_{l}$ of three selected cases with increasing $\theta_{2}$ before, at, and after the peak of $J_{a} / J_{o}$, as labeled in Fig. 14(a). Once again, we find a familiar variation of $\phi_{l}$ at the hopper exit, similar to those shown in Fig. 3(b), Fig. 6(b2), Fig. 10(a2, b1), and in our previous study [14]. The value of $\phi_{l}$ at the hopper exit when a peak of $J_{a} / J_{o}$ occurs is between 0.5 and 0.6 in all studied cases.

\section{CONCLUSIONS}

We investigate the phenomenon of the flow rate enhancement in a hopper discharging athermal granular particles passing an obstacle placed near its exit. Several competing mechanisms, such as interparticle collaborative motion and particle acceleration due to gravity are potentially responsible for the phenomenon. To decompose these competing mechanisms, we leverage a probabilistic 2D Tetris-like model, where particles move without creating overlaps between objects in the system by following a position-update algorithm without Newton's equations of motion. Our model preserves only the minimal dynamics necessary to investigate particle motion within the free space below the obstacle with or without acceleration due to an external driving force, such as gravity.

The enhanced flow rate phenomenon still occurs in our probabilistic model that switches off interparticle collaborative motion. We find that the peaking phenomenon is limited to a system of at least a few hundred of particles that pass through the channels between the obstacle and the hopper walls, and then concentrate below the obstacle on their way out of the hopper. Adjusting the height of the obstacle in the hopper can reduce the amount of particles passing through the channels between the obstacle and hopper walls and in turn decrease the particle packing fraction at the hopper exit. Particles of lower packing fraction exited the hopper faster. The flow rate enhancement can be explained by the above mechanism if an obstacle is placed at an optimal distance away from the hopper exit that mildly reduces the particle packing fraction in exchange for a faster discharging velocity. In all studied cases, the value of the local area packing frac- 

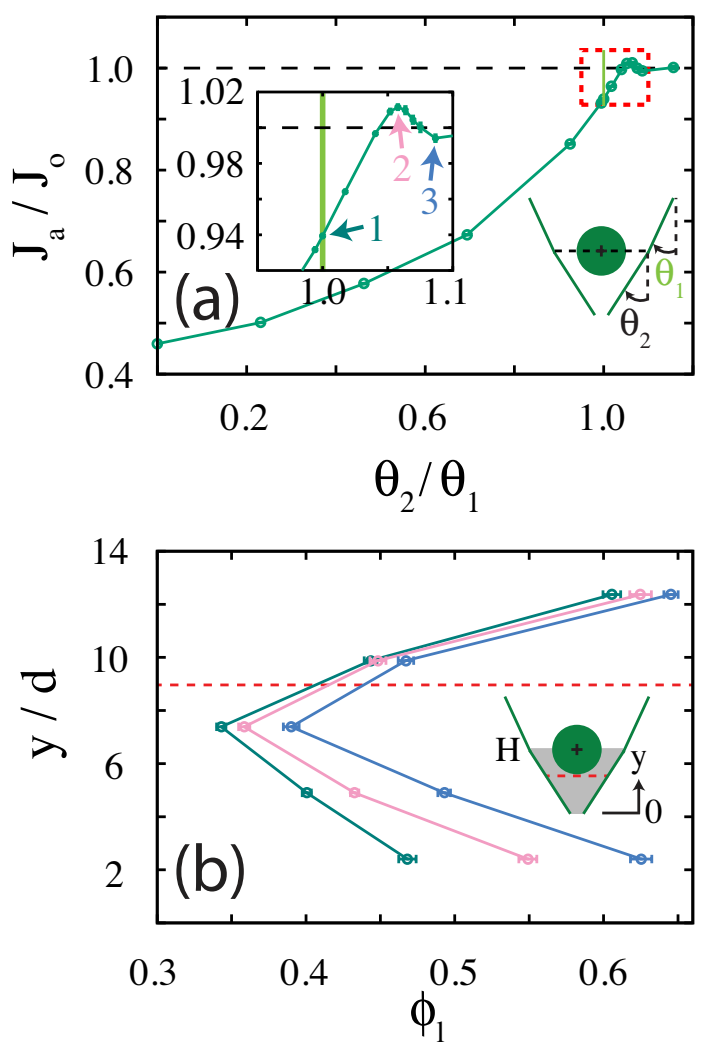

FIG. 14. (Color online) (a) The same plot as Fig. 13 except $\alpha_{y}=0.439$. (b) Averaged local area packing fraction $\phi_{l}$ in the shaded zone in-between $y=[0, H]$, as shown in the inset, of the three selected cases labeled in the inset of (a), where the value of $\theta_{2}$ is at 1 (dark green), 2 (pink), and 3 (navy). The horizontal dashed line indicates the lowest y position of the obstacle.

tion $\phi_{l}$ at the hopper exit when a flow rate peak occurs is within a range of 0.5 and 0.6 , which corresponds to the characteristic transition from $\phi_{l}^{E}<\phi_{o}^{c}$ to $\phi_{l}^{E}>\phi_{o}^{c}$, where $\phi_{o}^{c} \approx 0.55$ is a characteristic area packing fraction for the parameters chosen in this study. Too small $\phi_{l}$ implies ineffective particle concentration mechanism, while too large $\phi_{l}$ suggests interparticle repelling/clogging which are both unfavorable to enhance the flow rate. The char- acteristic transition suggests that particles unable to update their positions form a cluster (two particles near to each other are treated as clustered) that spans the hopper exit, as discussed in the appendix, Sec. V.

Based on our findings, we then utilized the concentration mechanism by artificially requiring particles below the obstacle to preferably move away from the hopper walls in the horizontal direction. We proved that this strategy can further amplify the value of the flow rate peak of weakly-driven particles or even create one in a relatively strongly-driven system that originally exhibits no peaking phenomenon. This confirms that the particle concentration mechanism at the hopper exit is indeed responsible for the flow rate enhancement. Although artificial, it is possible to test the anisotropic merging strategy experimentally using granular particles guided by engraved guidelines on a 2D hopper surface or using metallic particles responding to an external magnetic field 22 . Adding a shortcut path through the obstacle which allows particles to directly move towards the hopper exit interferes with the particle concentration mechanism and generally degrades the enhanced flow rate phenomenon.

Finally, we investigate the factors related to the waiting room effect by decomposing it into three parts: the void space below the obstacle, particle speed-up rate, and the exit geometry of the hopper. The waiting room effect, wherein particles are slowed down by the obstacle and then speed up within the void below it, is believed to contribute to the higher flow rate observed in the peaking phenomenon. Our results show that a flow rate peak still exists even when the defined void space of the waiting room has been reduced to zero and, more importantly, can be explained by the concentration mechanism. Moreover, the enhanced flow rate can be augmented even further if the channels between the obstacle and the hopper walls have a shrinking geometry towards the hopper exit. There also exists a narrow range of particle speed-up rate or hopper exit angles by which an existing flow rate peak can be further improved. The latter can also be utilized to create a flow rate peak manually.

All of these results support the conclusion that the concentration mechanism at the hopper exit alone can create the hopper flow rate enhancement, even if the waiting room effect is eliminated. However, in an experimental setup containing frictional granular particles, the concentration mechanism and the waiting room effect likely coexist, and in tandem give rise to the flow rate peaking phenomenon. We hope the results of our Tetris-like model can motivate theoretical work. We believe the particle concentration mechanism can be used for designing hoppers that discharge granular particles more efficiently and expect there to be broad industrial applications. 

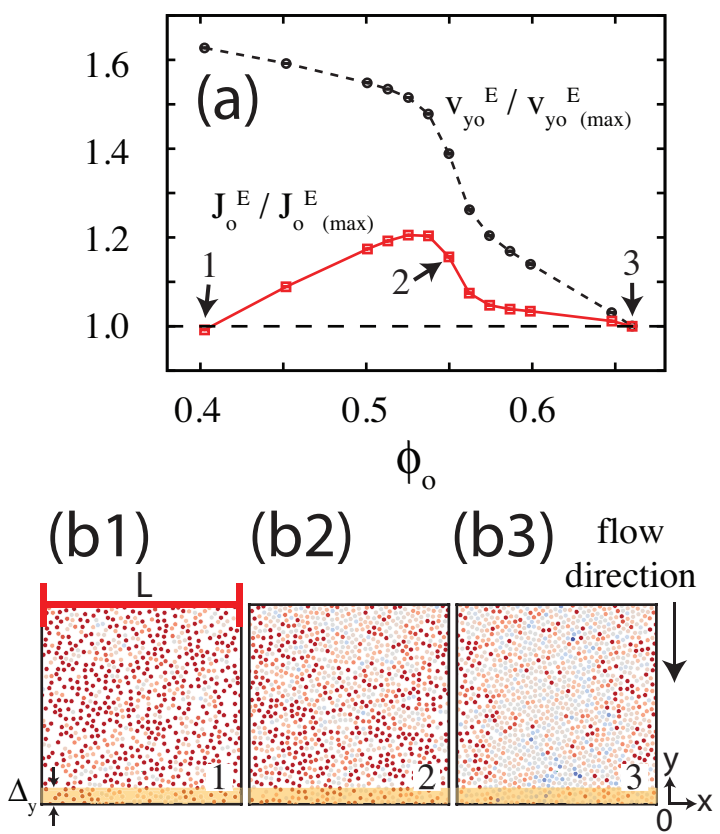

FIG. 15. (Color online) (a) Averaged particle velocity $v_{y o}^{E}$ (black circles), measured at $y_{c}=0.03125 L=1.25 d$ and normalized by $v_{y o(\max )}^{E}$ at $\phi_{o(\max )} \approx 0.66$, in a square hopper of side $L$ and a hopper angle $\theta_{1}=0$. The calculated normalized flow rate (red squares) is obtained using $J_{o}^{E} / J_{o(\max )}^{E}=\left(v_{y o}^{E} \phi_{o}\right) /\left(v_{y o(\max )}^{E} \phi_{o(\max )}\right)$. A sudden slowdown of $v_{y o}^{E}$ which creates a local peak of $J_{o}^{E}$ occurs when $\phi_{o}>\phi_{o}^{c} \approx 0.55$. Particles discharged from the hopper reenter it from its top boundary (red horizontal line in (b1)) with their $\mathrm{x}$ positions randomized. (b1-b3) Representative snapshots of the system as Fig. 4. except for the three cases indicated by arrows in (a), and $\Delta_{y}=0.0625 L=2.5 d$. Each mean and its error bar are obtained using 10 trials.

\section{APPENDIX: GENERAL FLOWING PROPERTY OF TETRIS PARTICLES}

In all studied cases, we observe that the range of local $\phi_{l}$ at the hopper exit is between 0.5 and 0.6 when a peak of $J_{a} / J_{o}$ occurs. To understand if this specific range of $\phi_{l}$ is related to a more intrinsic property of Tetris parti- cles without the influence of an obstacle or the shrinking hopper geometry, we chose a square hopper containing no obstacle with a hopper angle $\theta_{1}=0$, equivalent to a channel of constant width. In this setup, we can define a global area packing fraction $\phi_{o}$ as the ratio of the total area of particles in the hopper to its size. We measure the averaged particle velocity $v_{y o}^{E}$ at the hopper exit and calculate the corresponding flow rate $J_{o}^{E}=\phi_{o} v_{y o}^{E} w^{E}$, where $w^{E}=L$ is the constant hopper width. Both quantities are normalized by their values at $\phi_{o}(\max ) \approx 0.66$. The results are shown in Fig. 15.

In Fig. 15(a), we can see that $v_{y o}^{E}$ exhibits a sudden slow-down when $\phi_{o}$ exceeds a characteristic area packing fraction $\phi_{o}^{c} \approx 0.55$. Simultaneously, $J_{o}^{E}$ shows a peak right before $\phi_{o}>\phi_{o}^{c}$. In Fig. 15(b1) - (b3), we show the representative snapshots of the three selected cases before, at, and after $\phi_{o}^{c}$, labeled as 1, 2, and 3 in (a). Particles are colored in blue by $n^{f}$ or in red by $n^{s}$ according to their consecutive failed or successful position-update history. When $\phi_{o}<\phi_{o}^{c}$, we can see that particles unable to update their positions (with a nonzero $n^{f}$ ) form small and local clusters randomly distributed in the hopper. On the other hand, when $\phi_{o}>\phi_{o}^{c}$, particles of nonzero $n^{f}$ form a cluster across the system, especially along the flow direction. The phenomenon of the sudden slow-down due to an increasing $\phi_{o}$, and the ensuing frequent failures of particle position-update and system size comparable clustering behavior seems to be similar to the transition to slow dynamics where touching/nearly touching particles form a network in a system governed by Newtonian dynamics [23, 24]. An investigation to fully quantify this phenomenon of Tetris particles is left for future exploration.

\section{ACKNOWLEDGMENTS}

We thank Corey S. O'Hern for useful discussions. GJG acknowledges financial support from National Taiwan University funding 104R7417, MOST Grant No. 104-2218-E-002-019 (Taiwan), and computational facility made available by the startup funding of Shizuoka University (Japan).
[1] I. Zuriguel, A. Janda, A. Garcimartín, C. Lozano, R. Arévalo, and D. Maza, Phys. Rev. Lett. 107, 278001 (2011).

[2] I. Zuriguel, D. R. Parisi, R. C. Hidalgo, C. Lozano, A. Janda, P. A. Gago, J. P. Peralta, L. M. Ferrer, L. A. Pugnaloni, E. Clément, D. Maza, I. Pagonabarraga, and A. Garcimartín, Sci. Rep. 4, 7324 (2014).

[3] C. Lozano, A. Janda, A. Garcimartín, D. Maza, and I. Zuriguel, Phys. Rev. E 86, 031306 (2012).

[4] F. Alonso-Marroquin, S. I. Azeezullah, S. A. GalindoTorres, and L. M. Olsen-Kettle, Phys. Rev. E 85, 020301 (2012).
[5] J. M. Pastor, A. Garcimartín, P. A. Gago, J. P. P. amd César Martín-Gómez, L. M. Ferrer, D. Maza, D. R. Parisi, L. A. Pugnaloni, and I. Zuriguel, Phys. Rev. E 92, 062817 (2015).

[6] A. Murray and F. Alonso-Marroquin, Paper in Physics 8, 080003 (2016).

[7] D. Helbing, I. Farkas, and T. Vicsek, Nature 407, 487 (2000).

[8] R. Escobar and A. D. L. Rosa, Advances in Artificial Life (Springer, Berlin, 2003) pp. 97-106.

[9] D. Helbing, L. Buzna, A. Johansson, and T. Werner, Transp. Sci. 39, 1 (2005). 
[10] A. Garcimartín, J. M. Pastor, L. M. Ferrer, J. J. Ramos, C. Martín-Gómez, and I. Zuriguel, Phys. Rev. E 91, 022808 (2015).

[11] I. Zuriguel, J. Olivares, J. M. Pastor, C. Martín-Gómez, L. M. Ferrer, J. J. Ramos, and A. Garcimartín, Phys. Rev. E 94, 032302 (2016).

[12] K. Endo and H. Katsuragi, EPJ Web Conf. 140, 03004 (2017).

[13] K. Endo, K. A. Reddy, and H. Katsuragi, Phys. Rev. Fluids 2, 094302 (2018).

[14] G. J. Gao, J. Blawzdziewicz, M. C. Holcomb, and S. Ogata, Granul. Matter. 21: 25 (2019).

[15] G. J. Gao, J. Phys. Soc. Jpn. 87, 114401 (2018).

[16] E. Caglioti, V. Loreto, H. J. Herrmann, and M. Nicodemi, Phys. Rev. Lett. 79, 1575 (1997).
[17] A. Clauset, C. R. Shalizi, and M. E. J. Newman, SIAM Review 51, 661 (2009).

[18] E. Gardel, E. Seitaridou, K. Facto, E. Keene, K. Hattam, N. Easwar, and N. MENON, Phil. Trans. R. Soc. A 367, 5109 (2009).

[19] S. Tewari, M. Dichter, and B. Chakraborty, Soft Matter 9, 5016 (2013).

[20] C. C. Thomas and D. J. Durian, Phys. Rev. E 94, 022901 (2016).

[21] I. Zuriguel, D. Maza, A. Janda, R. C. Hidalgo, and A. Garcimartín, Granul. Matter. 21: 47 (2019).

[22] D. Hernández-Enríquez, G. Lumay, and F. PachecoVázquez, EPJ Web Conf. 140, 03089 (2017).

[23] T. Shen, C. Schreck, B. Chakraborty, D. E. Freed, and C. S. O'Hern, Phys. Rev. E 86, 041303 (2012).

[24] L. Papadopoulos, M. A. Porter, K. E. Daniels, and D. S. Bassett, J. Complex Netw. 6, 485 (2018). 